\title{
A PROBLEM ON ROUGH PARAMETRIC MARCINKIEWICZ FUNCTIONS
}

\author{
YONG DING, SHANZHEN LU and KôZÔ YABUTA
}

(Received 31 March 2000; revised 12 December 2000)

Communicated by A. H. Dooley

\begin{abstract}
In this note the authors give the $L^{2}\left(\mathbb{R}^{n}\right)$ boundedness of a class of parametric Marcinkiewicz integral $\mu_{\Omega, h}^{\rho}$ with kernel function $\Omega$ in $L \log ^{+} L\left(S^{n-1}\right)$ and radial function $h(|x|) \in l^{\infty}\left(L^{q}\right)\left(\mathbb{R}_{+}\right)$for $1<q \leq \infty$.

As its corollary, the $L^{p}\left(\mathbb{R}^{n}\right)(2 \leq p<\infty)$ boundedness of $\mu_{\Omega, h, \lambda}^{*, \rho}$ and $\mu_{\Omega, h, S}^{\rho}$ with $\Omega$ in $L \log ^{+} L\left(S^{n-1}\right)$ and $h(|x|) \in l^{\infty}\left(L^{q}\right)\left(\mathbb{R}_{+}\right)$are also obtained. Here $\mu_{\Omega, h, \lambda}^{*, \rho}$ and $\mu_{\Omega, h . S}^{\rho}$ are parametric Marcinkiewicz functions corresponding to the Littlewood-Paley $g_{\lambda}^{*}$-function and the Lusin area function $S$, respectively.

2000 Mathematics subject classification: primary 42B25, 42B99.

Keywords and phrases: Marcinkiewicz integral,Littlewood-Paley $g$-function, Lusin area integral, rough kernel.
\end{abstract}

\section{Introduction}

Suppose that $S^{n-1}$ is the unit sphere of $\mathbb{R}^{n}(n \geq 2)$ equipped with normalized Lebesgue measure $d \sigma=d \sigma\left(x^{\prime}\right)$. Let $\Omega \in L^{1}\left(S^{n-1}\right)$ be homogeneous of degree zero on $\mathbb{R}^{n}$ and

$$
\int_{S^{n-1}} \Omega\left(x^{\prime}\right) d \sigma\left(x^{\prime}\right)=0,
$$

where $x^{\prime}=x /|x|$ for any $x \neq 0$.

In 1960, Hörmander [5] defined the parametric Marcinkiewicz function of higher dimension as follows.

$$
\mu_{\Omega}^{\rho}(f)(x)=\left(\int_{0}^{\infty}\left|F_{t}^{\rho}(x)\right|^{2} \frac{d t}{t}\right)^{1 / 2}
$$

The first author and the second author were supported by NSF of China (Grant No. 19971010) and National 973 Project of China, respectively.

(C) 2002 Australian Mathematical Society 0263-61 15/2002 \$A2.00+0.00 
where $\rho>0$ and

$$
F_{t}^{\rho}(x)=\frac{1}{t^{\rho}} \int_{|x-y| \leq t} \frac{\Omega(x-y)}{|x-y|^{n-\rho}} f(y) d y .
$$

When $\rho=1$, we denote $\mu_{\Omega}^{1}$ simply by $\mu_{\Omega}$. It is well known that $\mu_{\Omega}$ is the usual Marcinkiewicz integral corresponding to the Littlewood-Paley $g$-function introduced by Stein in [7]. Stein proved that if $\Omega$ is continuous and $\Omega \in \operatorname{Lip}_{\alpha}\left(S^{n-1}\right)(0<\alpha \leq 1)$, then $\mu_{\Omega}$ is of type $(p, p)(1<p \leq 2)$ and of weak type $(1,1)$. In [1], Benedek, Calderón and Panzone proved that if $\Omega \in C^{1}\left(S^{n-1}\right)$, then $\mu_{\Omega}$ is of type $(p, p)(1<$ $p<\infty)$. On the other hand, in 1960, Hörmander [5] proved that if $\Omega \in \operatorname{Lip}_{\alpha}\left(S^{n-1}\right)$ $(0<\alpha \leq 1)$, then for $\rho>0, \mu_{\Omega}^{o}$ is of type $(p, p)(1<p<\infty)$. Recently, Sakamoto and Yabuta [6] gave the $L^{p}$ boundedness of $\mu_{\Omega}^{\rho}, \mu_{\Omega, \lambda}^{*, \rho}$ and and $\mu_{\Omega, S}^{\rho}$ (see below for the definitions), where $\rho$ is a complex number and $\Omega \in \operatorname{Lip}_{\alpha}\left(S^{n-1}\right)(0<\alpha \leq 1)$. It is worth pointing out that $\Omega$ was required to satisfy some smoothness conditions in the results mentioned above.

For a long time, an open problem is whether there are some results as above on the $L^{p}$ boundedness of parametric Marcinkiewicz function $\mu_{\Omega}^{\rho}$ when $\Omega$ satisfies only some size condition. The purpose of this note is to give a positive answer. Precisely, we shall consider $L^{2}\left(\mathbb{R}^{n}\right)$ boundedness of a class of parametric Marcinkiewicz function with kernel functions which lacks smoothness both on the sphere and in radial direction. Let us give some definitions first. The function spaces $l^{\infty}\left(L^{q}\right)\left(\mathbb{R}_{+}\right)$are defined as follows. If $1 \leq q<\infty$,

$$
l^{\infty}\left(L^{q}\right)\left(\mathbb{R}_{+}\right)=\left\{h:\|h\|_{l^{\infty}\left(L^{q}\right)\left(\mathbb{R}_{+}\right)}=\sup _{j \in \mathbb{Z}}\left(\int_{\mathcal{L}^{j-1}}^{2^{j}}|h(r)|^{q} \frac{d r}{r}\right)^{1 / q}<C\right\} .
$$

If $q=\infty, l^{\infty}\left(L^{\infty}\right)\left(\mathbb{R}_{+}\right)=L^{\infty}\left(\mathbb{R}_{+}\right)$. By Hölder's inequality, it is easy to check that for $1<q<r<\infty$

$$
l^{\infty}\left(L^{\infty}\right) \subset l^{\infty}\left(L^{r}\right) \subset l^{\infty}\left(L^{q}\right) \subset l^{\infty}\left(L^{1}\right) .
$$

The parametric Marcinkiewicz function $\mu_{\Omega, h}^{\rho}$ is defined by

$$
\mu_{\Omega, h}^{\rho}(f)(x)=\left(\int_{0}^{\infty}\left|F_{\Omega, h}^{\rho}(x, t)\right|^{2} \frac{d t}{t}\right)^{1 / 2},
$$

where $\rho$ is a complex number, $\rho=\alpha+i \tau$ and $h(x)$ is a radial function on $\mathbb{R}^{n}$ satisfying $h(|x|) \in l^{\infty}\left(L^{q}\right)\left(\mathbb{B}_{+}\right)(1 \leq q \leq \infty)$,

$$
F_{\Omega, h}^{\rho}(x, t)=\frac{1}{t^{\rho}} \int_{|x-y| \leq t} \frac{\Omega(x-y) h(|x-y|)}{|x-y|^{n-\rho}} f(y) d y .
$$

Our main result is the following theorem. 
THEOREM 1. Suppose that $\Omega \in L \log ^{+} L\left(S^{n-1}\right)$ is a homogeneous function of degree zero on $\mathbb{R}^{n}$ satisfying (1.1) and $h(|x|) \in l^{\infty}\left(L^{q}\right)\left(\mathbb{R}_{+}\right)$. If $1<q \leq \infty$ and $\operatorname{Re}(\rho)=\alpha>0$, then $\left\|\mu_{\Omega, h}^{\rho}(f)\right\|_{2} \leq C / \sqrt{\alpha}\|f\|_{2}$, where $C$ is independent of $\rho$ and $f$.

As an application of Theorem 1, we obtain also the $L^{p}\left(\mathbb{R}^{n}\right)(p \geq 2)$ boundedness of the parametric Marcinkiewicz functions $\mu_{\Omega, h, \lambda}^{*, \rho}$ and $\mu_{\Omega, h, S}^{\rho}$ with the same kernel function $\Omega$ and $h(x)$, where $\mu_{\Omega, h, \lambda}^{*, \rho}$ and $\mu_{\Omega, h, S}^{\rho}$ are respectively defined by

$$
\begin{aligned}
& \mu_{\Omega, h, \lambda}^{*, \rho}(f)(x)=\left(\iint_{\mathbb{R}_{+}^{n+1}}\left(\frac{t}{t+|x-y|}\right)^{n \lambda}\left|F_{\Omega, h}^{\rho}(y, t)\right|^{2} \frac{d y d t}{t^{n+1}}\right)^{1 / 2}, \lambda>1, \\
& \mu_{\Omega, h, S}^{\rho}(f)(x)=\left(\int_{\Gamma(x)}\left|F_{\Omega, h}^{\rho}(y, t)\right|^{2} \frac{d y d t}{t^{n+1}}\right)^{1 / 2},
\end{aligned}
$$

where $\Gamma(x)=\left\{(y, t) \in \mathbb{R}_{+}^{n+1}:|x-y|<t\right\}$.

THEOREM 2. If $2 \leq p<\infty$, then under the conditions of Theorem 1 we have $\left\|\mu_{\Omega, h, \lambda}^{*, \rho}(f)\right\|_{p} \leq(C / \sqrt{\alpha})\|f\|_{p}$ and $\left\|\mu_{\Omega, h, S}^{\rho}(f)\right\|_{p} \leq(C / \sqrt{\alpha})\|f\|_{p}$, where $C=C_{\lambda, n, p}$ is independent of $\rho$ and $f$.

REMARK 1. Note that

$$
\begin{aligned}
\operatorname{Lip}_{\alpha}\left(S^{n-1}\right)(0<\alpha \leq 1) \subset L^{\infty}\left(S^{n-1}\right) \subset L^{q}\left(S^{n-1}\right)(q>1) \\
\subset L \log ^{+} L\left(S^{n-1}\right) \subset L^{1}\left(S^{n-1}\right),
\end{aligned}
$$

and all inclusions are proper. Therefore in Theorem 1 and Theorem 2, the smoothness condition assumed on $\Omega$ has been removed and Theorem 1 and Theorem 2 are improvement and extension of the known results mentioned above for $p=2$ and $2 \leq p<\infty$, respectively.

REMARK 2. After finishing this paper, we were told that in recent work [4], using a method which is quite different from one in this paper, Fan and Sato also gave the $L^{2}$-boundedness of Marcinkiewicz integral $\mu_{\Omega}^{o}$ when $\Omega \in L \log ^{+} L\left(S^{n-1}\right)$ and $h \equiv 1$. From their result, one can deduce that $\left(L^{2}, L^{2}\right)$ bound of $\mu_{\Omega}^{\rho}$ is only smaller than $C\left((\operatorname{Re} \rho)^{-3 / 2}+(\operatorname{Re} \rho)^{-1 / 2}\right)$. However, it is smaller than $C(\operatorname{Re} \rho)^{-1 / 2}$ by our method. Hence the conclusion of Theorem 1 in this paper is better than the relevant result in [4].

\section{Proofs of Theorem 1 and Theorem 2}

Let us begin by recalling a known fact. 
LEMMA 1. Let $\Omega\left(x^{\prime}\right) \in L^{\infty}\left(S^{n-1}\right)$. Then for any $0<\theta<1$ there is a constant $C$ such that for all $j \in \mathbb{Z}$,

$$
\left(\int_{2^{j}}^{2^{j+1}}\left|\int_{S^{n-1}} \Omega\left(u^{\prime}\right) e^{-2 \pi i r u^{\prime} \cdot x} d \sigma\left(u^{\prime}\right)\right|^{2} \frac{d r}{r}\right)^{1 / 2} \leq C\|\Omega\|_{L^{\infty}\left(S^{n-1}\right)}\left|2^{j} x\right|^{-\theta / 2} .
$$

See [3] for the proof.

LEMMA 2. Let $\Omega\left(x^{\prime}\right) \in L^{\infty}\left(S^{n-1}\right)$ and $h(r) \in l^{\infty}\left(L^{q}\right)\left(\mathbb{R}_{+}\right), 1 \leq q \leq 2$. Then for any $0<\theta<1$ there is a constant $C$ such that for all $j \in \mathbb{Z}$,

$$
\begin{aligned}
& \int_{2^{j}}^{2^{j+1}}\left|\int_{S^{n-1}} \Omega\left(u^{\prime}\right) e^{-2 \pi i r u^{\prime} \cdot x} h(r) d \sigma\left(u^{\prime}\right)\right| \frac{d r}{r} \\
& \left.\quad \leq C\|h\|_{L^{\infty}\left(L^{q}\right)\left(\mathbb{R}_{+}\right)}\left(\|\Omega\|_{L^{\infty}\left(S^{n-1}\right)}\left|2^{j} x\right|^{-\theta / 2}\right)^{2 / q^{\prime}}\left(\|\Omega\|_{L^{\prime}\left(S^{n-1}\right)}\right)\right)^{\left(q^{\prime}-2\right) / q^{\prime}} .
\end{aligned}
$$

Proof. Denote by

$$
K(h)=\int_{2}^{2^{j+1}}\left|\int_{S^{n-1}} \Omega\left(u^{\prime}\right) e^{-2 \pi i r u^{\prime} \cdot x} h(r) d \sigma\left(u^{\prime}\right)\right| \frac{d r}{r} .
$$

First let us consider the case $q=2$. By Lemma 1 and Hölder's inequality we obtain

$$
\begin{aligned}
K(h) & \leq\|h\|_{l^{\infty}\left(L^{2}\right)\left(\mathbb{R}_{+}\right)}\left(\int_{2^{j}}^{2^{j+1}}\left|\int_{S^{n-1}} \Omega\left(u^{\prime}\right) e^{-2 \pi i r u^{\prime} \cdot x} d \sigma\left(u^{\prime}\right)\right|^{2} \frac{d r}{r}\right)^{1 / 2} \\
& \leq C\|h\|_{l^{\infty}\left(L^{2}\right)\left(\mathbb{R}_{+}\right)}\|\Omega\|_{L^{\infty}\left(S^{n-1}\right)}\left|2^{j} x\right|^{-\theta / 2} .
\end{aligned}
$$

On the other hand, for $q=1$ we have

$$
K(h) \leq \int_{2^{j}}^{2^{j+1}} \int_{S^{n-1}}\left|\Omega\left(u^{\prime}\right)\right| d \sigma\left(u^{\prime}\right)|h(r)| \frac{d r}{r} \leq\|h\|_{L^{\infty}\left(L^{\prime}\right)\left(\mathbf{R}_{+}\right)}\|\Omega\|_{L^{\prime}\left(S^{n-1}\right)} .
$$

Hence if we see $K$ as a sublinear operator acted on the spaces $l^{\infty}\left(L^{q}\right)\left(\mathbb{R}_{+}\right)$for $1 \leq q \leq 2$, then (2.2) and (2.3) show that $K$ is a bounded operator from $l^{\infty}\left(L^{2}\right)\left(\mathbb{B}_{+}\right)$ to $L^{\infty}$ and from $l^{\infty}\left(L^{1}\right)\left(\mathbb{R}_{+}\right)$to $L^{\infty}$, respectively. Using the Riesz-Thorin interpolation theorem for sublinear operator [2] between (2.2) and (2.3), we know there exists an $\eta$ satisfying $0<\eta<1$ and $1 / q=(1-\eta)+\eta / 2$ such that

$$
K(h) \leq C\|h\|_{\mathcal{L}^{\infty}\left(L^{q}\right)\left(\mathbb{R}_{+}\right)}\left(\|\Omega\|_{L^{\infty}\left(S^{n-1}\right)}\left|2^{j} x\right|^{-\theta / 2}\right)^{\eta}\left(\|\Omega\|_{L^{1}\left(S^{n-1}\right)}\right)^{1-\eta} .
$$

It is easy to see that $\eta=2 / q^{\prime}$. Thus we finish the proof of Lemma 2 .

Now let us turn to the proof of Theorem 1 . 
ProOF OF THEOREM 1. By (1.3) we need only consider the case $1<q \leq 2$. First we have

$$
\hat{F}_{\Omega, h}^{\rho}(\xi, t)=\int_{\mathbb{R}^{n}} e^{-2 \pi i x \cdot \xi} F_{\Omega, h}^{\rho}(x, t) d x=\hat{f}(\xi) \frac{1}{t^{\rho}} \int_{|u| \leq t} \frac{\Omega(u) h(|u|)}{|u|^{n-\rho}} e^{-2 \pi i u \cdot \xi} d u
$$

Using Plancherel's theorem and (2.4), the square of $L^{2}\left(\mathbb{R}^{n}\right)$-norm of $\mu_{\Omega, h}^{\rho}(f)$ is equal to

$$
\int_{0}^{\infty} \int_{\mathbb{R}^{n}}\left|\hat{F}_{\Omega, h}^{\rho}(\xi, t)\right|^{2} d \xi \frac{d t}{t}=\int_{\mathbb{R}^{n}}|\hat{f}(\xi)|^{2}\left(\int_{0}^{\infty}\left|\int_{|u| \leq t} \frac{\Omega(u) h(|u|)}{|u|^{n-\rho}} e^{-2 \pi i u \cdot \xi} d u\right|^{2} \frac{d t}{t^{1+2 \alpha}}\right) d \xi .
$$

Since

$$
\begin{aligned}
\left(\int_{0}^{\infty} \mid\right. & \left.\left|\int_{|u| \leq t} \frac{\Omega(u) h(|u|)}{|u|^{n-\rho}} e^{-2 \pi i u^{\prime} \cdot} d u\right|^{2} \frac{d t}{t^{1+2 \alpha}}\right)^{1 / 2} \\
\quad & \left(\int_{0}^{\infty}\left|\int_{0}^{\infty} \int_{S^{n-1}} \Omega\left(u^{\prime}\right) e^{-2 \pi i r u^{\prime} \cdot \xi} \frac{\chi_{[0, t]}(r)}{r^{1-\rho}} h(r) d \sigma\left(u^{\prime}\right) d r\right|^{2} \frac{d t}{t^{1+2 \alpha}}\right)^{1 / 2} \\
& \leq \int_{0}^{\infty}\left(\int_{0}^{\infty}\left|\int_{S^{n-1}} \Omega\left(u^{\prime}\right) e^{-2 \pi i r u^{\prime} \cdot \xi} h(r) d \sigma\left(u^{\prime}\right)\right|^{2} \frac{\chi_{[0, t]}(r)}{t^{1+2 \alpha}} d t\right)^{1 / 2} \frac{d r}{r^{1-\alpha}} \\
& =\int_{0}^{\infty}\left|\int_{S^{n-1}} \Omega\left(u^{\prime}\right) e^{-2 \pi i r u^{\prime} \cdot \xi} h(r) d \sigma\left(u^{\prime}\right)\right|\left(\int_{r}^{\infty} \frac{d t}{t^{1+2 \alpha}}\right)^{1 / 2} \frac{d r}{r^{1-\alpha}} \\
& =\frac{1}{\sqrt{2 \alpha}} \int_{0}^{\infty}\left|\int_{S^{n-1}} \Omega\left(u^{\prime}\right) e^{-2 \pi i r u^{\prime} \cdot \xi} h(r) d \sigma\left(u^{\prime}\right)\right| \frac{d r}{r} .
\end{aligned}
$$

On the other hand, note that for any $s>0$, we have

$$
\left(\int_{2^{j-1}}^{2^{j}}|h(r s)|^{q} \frac{d r}{r}\right)^{1 / q}=\left(\int_{2^{-1} s}^{2^{j} s}|h(r)|^{q} \frac{d r}{r}\right)^{1 / q} \leq 2\|h\|_{l^{\infty}\left(L^{q}\right)\left(\mathbb{R}_{+}\right)} .
$$

Therefore, by (2.5) to prove Theorem 1 it suffices to show that for $\Omega \in L \log ^{+} L\left(S^{n-1}\right)$, there is a constant $C$ such that

$$
\sup _{x^{\prime} \in S^{n-1}} \int_{0}^{\infty}\left|\int_{S^{n-1}} \Omega\left(u^{\prime}\right) e^{-2 \pi i r u^{\prime} \cdot x^{\prime}} h(r) d \sigma\left(u^{\prime}\right)\right| \frac{d r}{r} \leq C .
$$

For any $x^{\prime} \in S^{n-1}$, we denote $G\left(x^{\prime}, r\right)=\int_{S^{n-1}} \Omega\left(u^{\prime}\right) e^{-2 \pi i r u^{\prime} \cdot x^{\prime}} d \sigma\left(u^{\prime}\right)$ and write

$$
\int_{0}^{\infty}\left|G\left(x^{\prime}, r\right) h(r)\right| \frac{d r}{r}=\int_{0}^{2}\left|G\left(x^{\prime}, r\right) h(r)\right| \frac{d r}{r}+\int_{2}^{\infty}\left|G\left(x^{\prime}, r\right) h(r)\right| \frac{d r}{r}=: \mathrm{I}+\mathrm{II} .
$$


Below we shall show that $I$ and $I I$ are uniformly bounded on $x^{\prime} \in S^{n-1}$. By (1.1), we have

$$
\mathrm{I}=\int_{0}^{2}\left|\int_{S^{n-1}} \Omega\left(u^{\prime}\right)\left(e^{-2 \pi i r u^{\prime} \cdot x^{\prime}}-1\right) h(r) d \sigma\left(u^{\prime}\right)\right| \frac{d r}{r} \leq C\|h\|_{L^{\infty}\left(L^{q}\right)\left(\mathbb{R}_{+}\right)}\|\Omega\|_{L^{\prime}\left(S^{n-1}\right)} .
$$

In order to give the estimate of II, we need to use some idea from [8]. Set

$$
\begin{aligned}
E_{0} & =\left\{u^{\prime} \in S^{n-1}:\left|\Omega\left(u^{\prime}\right)\right| \leq 2\right\}, \\
E_{l} & =\left\{u^{\prime} \in S^{n-1}: 2^{l}<\left|\Omega\left(u^{\prime}\right)\right| \leq 2^{l+1}\right\} \text { for } l \geq 1, \\
\Omega_{l}\left(u^{\prime}\right) & =\Omega\left(u^{\prime}\right) \chi_{E_{l}}\left(u^{\prime}\right) \text { for } l \geq 0, \\
G_{l}\left(x^{\prime}, r\right) & =\int_{S^{n-1}} \Omega_{l}\left(u^{\prime}\right) e^{-2 \pi i r u^{\prime} \cdot x^{\prime}} d \sigma\left(u^{\prime}\right) \text { for } l \geq 0,
\end{aligned}
$$

where $\chi_{E_{l}}\left(u^{\prime}\right)$ is the characteristic function of $E_{l}$. Taking $s \in \mathbb{N}$ such that $s \theta>q^{\prime}$, where $0<\theta<1$ is defined in Lemma 1. Then we have

$$
\begin{aligned}
\mathrm{II} & \leq \sum_{j=1}^{\infty} \int_{2^{j}}^{2^{j+1}}\left|G_{0}\left(x^{\prime}, r\right) h(r)\right| \frac{d r}{r}+\left(\sum_{l>0} \sum_{1 \leq j \leq s l}+\sum_{l>0} \sum_{j>s l}\right) \int_{2^{j}}^{2^{j+1}}\left|G_{l}\left(x^{\prime}, r\right) h(r)\right| \frac{d r}{r} \\
& =: \mathrm{II}_{1}+\mathrm{II}_{2}+\mathrm{II}_{3} .
\end{aligned}
$$

Now let us give the estimates for $\mathrm{II}_{1}, \mathrm{II}_{2}$ and $\mathrm{II}_{3}$, respectively. By Hölder's inequality

$$
\int_{2^{j}}^{2^{j+1}}\left|G_{0}\left(x^{\prime}, r\right) h(r)\right| \frac{d r}{r} \leq\|h\|_{l^{\infty}\left(L^{q}\right)\left(\mathbb{R}_{+}\right)}\left(\int_{\nu^{2 j+1}}^{2^{j+1}}\left|G_{0}\left(x^{\prime}, r\right)\right|^{q^{\prime}} \frac{d r}{r}\right)^{1 / q^{\prime}} .
$$

Since $\left|G_{0}\left(x^{\prime}, r\right)\right| \leq 2\left|S^{n-1}\right|$ and $2 \leq q^{\prime}<\infty$, by (2.8) we have

$$
\begin{aligned}
\int_{2^{j}}^{2^{j+1}} & \left|G_{0}\left(x^{\prime}, r\right) h(r)\right| \frac{d r}{r} \\
& \leq C\|h\|_{l^{\infty}\left(L^{q}\right)\left(\mathbf{R}_{+}\right)}\left(\int_{2^{j}}^{2^{j+1}}\left|G_{0}\left(x^{\prime}, r\right)\right|^{2}\left|G_{0}\left(x^{\prime}, r\right)\right|^{q^{\prime}-2} \frac{d r}{r}\right)^{1 / q^{\prime}} \\
& \leq C\|h\|_{l^{\infty}\left(L^{q}\right)\left(\mathbb{R}_{+}\right)}\left(\int_{\mathcal{V}^{j}}^{2^{j+1}}\left|G_{0}\left(x^{\prime}, r\right)\right|^{2} \frac{d r}{r}\right)^{1 / q^{\prime}} .
\end{aligned}
$$

By Lemma 1 and (2.9) we see that

$$
\mathrm{II}_{1}=\sum_{j=1}^{\infty} \int_{2^{j}}^{2^{j+1}}\left|G_{0}\left(x^{\prime}, r\right) h(r)\right| \frac{d r}{r}
$$




$$
\leq C\|h\|_{l^{\infty}\left(L^{q}\right)\left(\mathbb{R}_{+}\right)} \sum_{j=1}^{\infty}\left|2^{j} x^{\prime}\right|^{-\theta / q^{\prime}} \leq C\|h\|_{1^{\infty}\left(L^{q}\right)\left(\mathbb{R}_{+}\right)} .
$$

For $\mathrm{II}_{2}$ and $1<q \leq 2$ we obtain

$$
\begin{aligned}
\mathrm{II}_{2} & \leq \sum_{l>0} \sum_{1 \leq j \leq s l} \int_{2^{j}}^{2^{j+1}} \int_{S^{n-1}}\left|\Omega_{l}\left(u^{\prime}\right)\right| d \sigma\left(u^{\prime}\right)|h(r)| \frac{d r}{r} \\
& \leq C\|h\|_{l^{\infty}\left(L^{q}\right)\left(\mathbb{R}_{+}\right)} \sum_{l>0} \sum_{1 \leq j \leq s l}(\log 2)^{1 / q^{\prime}} \cdot\left\|\Omega_{l}\right\|_{L^{1}\left(S^{n-1}\right)} \\
& \leq C\|h\|_{l^{\infty}\left(L^{q}\right)\left(\mathbb{R}_{+}\right)} \sum_{l>0} l \log 2 \cdot 2^{l+1}\left|E_{l}\right| \leq C\|h\|_{l^{\infty}\left(L^{q}\right)\left(\mathbb{R}_{+}\right)}\|\Omega\|_{L \log ^{+} L\left(S^{n-1}\right)} .
\end{aligned}
$$

Finally, let us estimate $\mathrm{Il}_{3}$. Applying Lemma 2, we have

$$
\begin{aligned}
\mathrm{I}_{3} & =\sum_{l>0} \sum_{j>s l} \int_{2^{j}}^{2^{j+1}}\left\{\int_{S^{n-1}} \Omega_{l}\left(u^{\prime}\right) e^{-2 \pi i r u^{\prime} \cdot x^{\prime}} h(r) d \sigma\left(u^{\prime}\right) \mid \frac{d r}{r}\right. \\
& \left.\leq C\|h\|_{l^{\infty}\left(L^{q}\right)\left(\mathbb{R}_{+}\right)} \sum_{l>0} \sum_{j>s l}\left(\left\|\Omega_{l}\right\|_{L^{\infty}\left(S^{n-1}\right)} \mid 2^{j} x^{\prime}\right\}^{-\theta / 2}\right)^{2 / q^{\prime}}\left(\left\|\Omega_{l}\right\|_{L^{l}\left(S^{n-1}\right)}\right)^{\left(q^{\prime}-2\right) / q^{\prime}} \\
& \leq C\|h\|_{l^{\infty}\left(L^{q}\right)\left(\mathbb{R}_{+}\right)} \sum_{l>0} \sum_{j>s l}\left(2^{l} \cdot 2^{-j \theta / 2}\right)^{2 / q^{\prime}}\left(2^{l}\left|S^{n-1}\right|\right)^{\left(q^{\prime}-2\right) / q^{\prime}} \\
& \leq C\|h\|_{l^{\infty}\left(L^{q}\right)\left(\mathbb{R}_{+}\right)} \sum_{l>0} 2^{l} \cdot 2^{-s l \theta / q^{\prime}} \leq C\|h\|_{l^{\infty}\left(L^{q}\right)\left(\mathbb{R}_{+}\right)}
\end{aligned}
$$

It is easy to see that the constants in (2.7) and (2.10)-(2.12) are independent of $x^{\prime}$. Therefore, (2.6) follows from (2.7) and (2.10)-(2.12). Thus we complete the proof of Theorem 1.

Before giving the proof of Theorem 2, we give a lemma.

LEMMA 3. Let $\lambda>1$. Then under the conditions of Theorem 1 , there is a constant $C>0$ such that for any nonnegative and locally integrable function $\phi$,

$$
\left(\int_{\mathbb{R}^{n}} \mu_{\Omega, h, 2}^{*, \rho}(f)(x)^{2} \phi(x) d x\right)^{1 / 2} \leq \frac{C_{\lambda, n}}{\sqrt{\alpha}}\left(\int_{\mathbb{R}^{n}}|f(x)|^{2} M \phi(x) d x\right)^{1 / 2},
$$

where $M$ denotes the Hardy-Littlewood maximal operator.

The proof of Lemma 3 follows by using the method in [9, pages 241-242] and the conclusion of Theorem 1. We omit the details here. Now let us return to the proof of Theorem 2. 
ProOf OF THEOREM 2. For $2 \leq p<\infty$, we have

$$
\begin{aligned}
\left\|\mu_{\Omega, h, \lambda}^{*, \rho}(f)\right\|_{p} & =\left\{\left(\int_{\mathbb{R}^{n}}\left[\mu_{\Omega, h, \lambda}^{*, \rho}(f)(x)^{2}\right]^{p / 2} d x\right)^{2 / p}\right\}^{1 / 2} \\
& =\left\{\sup _{\phi}\left|\int_{\mathbb{R}^{n}} \mu_{\Omega, h, \lambda}^{*, \rho}(f)(x)^{2} \phi(x) d x\right|\right\}^{1 / 2},
\end{aligned}
$$

where the supremum is taken over all $\phi(x)$ satisfying $\|\phi\|_{(p / 2)^{\prime}} \leq 1$. Applying Lemma 3, Hölder's inequality and the $L^{(p / 2)^{\prime}}\left(1<(p / 2)^{\prime} \leq \infty\right)$ boundedness of Hardy-Littlewood maximal operator $M$, we get

$$
\left(\int_{\mathbb{R}^{n}} \mu_{\Omega, h, \lambda}^{*, \rho}(f)(x)^{2}|\phi(x)| d x\right)^{1 / 2} \leq \frac{C}{\sqrt{\alpha}}\left(\int_{\mathbb{R}^{n}}|f(x)|^{2} M \phi(x) d x\right)^{1 / 2} \leq \frac{C}{\sqrt{\alpha}}\|f\|_{p} .
$$

By (2.13) and (2.14) we have $\left\|\mu_{\Omega, h, \lambda}^{* . p}(f)\right\|_{p} \leq C / \sqrt{\alpha}\|f\|_{p}$. On the other hand, using the idea in [9] it is easy to prove that $\mu_{\Omega, h, S}^{\rho}(f)(x) \leq 2^{\lambda n} \mu_{\Omega, h, \lambda}^{*, \rho}(f)(x)$. Thus we complete the proof of Theorem 2.

Finally, we give another direct application of Lemma 3. It is well known that if $\omega \in A_{1}$, then $M \omega(x) \leq C \omega(x)$ a.e. on $\mathbb{R}^{n}$. Hence by Lemma 3, we get immediately the weighted $L^{2}$ boundedness for $\mu_{\Omega, h, \lambda}^{*, \rho}$ and $\mu_{\Omega, h, S}^{\rho}$.

COROLlaRY 1. Under the conditions of Theorem 1, if $\omega \in A_{1}$, then

$$
\left\|\mu_{\Omega, h, s}^{\rho}(f)\right\|_{2, \omega} \leq C_{\lambda, n}\left\|\mu_{\Omega, h, \lambda}^{*, \rho}(f)\right\|_{2, \omega} \leq \frac{C_{\lambda, n}}{\sqrt{\alpha}}\|f(x)\|_{2, \omega} .
$$

\section{References}

[1] A. Benedek, A. Calderón and R. Panzone, 'Convolution operators on Banach space valued functions', Proc. Nat. Acad. Sci. USA 48 (1962), 356-365.

[2] A. Calderón and A. Zygmund, 'A note on the interpolation of sublinear operators', Amer. J. Math. 78 (1956), 282-288.

[3] J. Duoandikoetxea and J. L. Rubio de Francia, 'Maximal and singular integral operators via Fourier transform estimates', Invent. Math. 84 (1986), 541-561.

[4] D. Fan and S. Sato, 'Weak type $(1,1)$ estimates for Marcinkiewicz integrals with rough kernels', Tôhoku Math. J., to appear.

[5] L. Hörmander, 'Translation invariant operators', Acta Math. 104 (1960), 93-139.

[6] M. Sakamoto and K. Yabuta, 'Boundedness of Marcinkiewicz functions', Studia Math. 135 (1999), 103-142. 
[7] E. M. Stein, 'On the functions of Littlewood-Paley, Lusin and Marcinkiewicz', Trans. Amer. Math. Soc. 88 (1958), 430-466.

[8] Q. Sun, 'Two problems about singular integral operators', Approx. Theory Appl. 7 (1991), 83-98.

[9] A. Torchinsky and S. Wang, 'A note on the Marcinkiewicz integral', Coll. Mat. 61-62 (1990), 235-243.

Department of Mathematics

School of Science

Beijing Normal University

Beijing, 100875

Kwansei Gakuin University

P. R. China

Uegahara 1-1-155

e-mail: dingy@bnu.edu.cn

Nishinomiya 662-8501

e-mail: lusz@bnu.edu.cn

Japan

e-mail: yabuta@kwansei.ac.jp 
